Journal for ImmunoTherapy of Cancer

\section{Changes in T-cell subsets and clonal repertoire during chemoimmunotherapy with pembrolizumab and paclitaxel or capecitabine for metastatic triple- negative breast cancer}

To cite: Chun B, Pucilowska J, Chang S, et al. Changes in T-cell subsets and clonal repertoire during chemoimmunotherapy with pembrolizumab and paclitaxel or capecitabine for metastatic triple-negative breast cancer. Journal for Immunotherapy of Cancer 2022;10:e004033. doi:10.1136/jitc-2021-004033

- Additional supplemental material is published online only. To view, please visit the journal online (http://dx.doi.org/10. 1136/jitc-2021-004033).

Accepted 07 December 2021

Check for updates

(c) Author(s) (or their employer(s)) 2022. Re-use permitted under CC BY-NC. No commercial re-use. See rights and permissions. Published by BMJ.

For numbered affiliations see end of article.

Correspondence to

Dr David B Page;

david.page2@providence.org

\section{ABSTRACT}

Background Chemoimmunotherapy is a standard treatment for triple-negative breast cancer (TNBC), however, the impacts of different chemotherapies on T-cell populations, which could correlate with clinical activity, are not known. Quantifying T-cell populations with flow cytometry and T-cell receptor (TCR) immunosequencing may improve our understanding of how chemoimmunotherapy affects T-cell subsets, and to what extent clonal shifts occur during treatment. TCR immunosequencing of intratumoral T cells may facilitate the identification and monitoring of putatively tumorreactive T-cell clones within the blood.

Methods Blood and tumor biopsies were collected from patients with metastatic TNBC enrolled in a phase $\mathrm{lb}$ clinical trial of first or second-line pembrolizumab with paclitaxel or capecitabine. Using identical biospecimen processing protocols, blood samples from a cohort of patients treated for early-stage breast cancer were obtained for comparison. Treatment-related immunological changes in peripheral blood and intratumoral T cells were characterized using flow cytometry and TCR immunosequencing. Clonal proliferation rates of $\mathrm{T}$ cells were compared based on intratumoral enrichment. Results When combined with pembrolizumab, paclitaxel and capecitabine resulted in similar time-dependent lymphodepletions across measured peripheral Tcell subsets. Their effects were more modest than that observed following curative-intent dose-dense anthracycline and cyclophosphamide (ddAC) (average fold-change in $\mathrm{CD}^{+}$cells, capecitabine: -0.42 , paclitaxel: -0.56 , ddAC: -1.21$)$. No differences in T-cell clonality or richness were observed following capecitabine or paclitaxel-based treatments. Regression modeling identified differences in the emergence of novel T-cell clones that were not detected at baseline (odds compared with ddAC, capecitabine: 0.292, paclitaxel: 0.652). Pembrolizumab with paclitaxel or capecitabine expanded T-cell clones within tumors; however, these clones did not always expand within the blood. Proliferation rates within the blood were similar between clones that were enriched and those that were not enriched within tumors.

Conclusion Chemoimmunotherapy for metastatic TNBC with pembrolizumab and capecitabine or paclitaxel resulted in similar peripheral T-cell subset lymphodepletion without altering T-cell clonal diversity. Regression modeling methods are applicable in immune monitoring studies, such as this to identify the odds of novel T-cell clones emerging during treatment, and proliferation rates of tumor-enriched T-cell clones.

\section{INTRODUCTION}

Recent phase III trials in metastatic triplenegative breast cancer (mTNBC) have demonstrated improved patient outcomes when chemotherapy was combined with antiprogrammed death 1/ligand 1 (anti-PD-1/L1) monoclonal antibodies. The IMpassion 130 study demonstrated improved progressionfree survival (PFS) with nab-paclitaxel and atezolizumab (PFS anti-PD-L1, HR=0.62, $\mathrm{p}<0.001)$, and the Keynote-355 study demonstrated an improved PFS and overall survival (OS) with pembrolizumab plus various chemotherapies (nab-paclitaxel, paclitaxel, or gemcitabine with carboplatin) (PFS antiPD-1, HR=0.65, $\mathrm{p}=0.0012) .^{1}{ }^{2}$ Despite these successes, not all chemoimmunotherapy regimens in all settings have improved outcomes for patients with metastatic disease. For example, neither first-line atezolizumab and paclitaxel nor later-line pembrolizumab and capecitabine improved OS or PFS, respectively. $^{34}$ These discordant responses could be due to differential immune effects of the chemotherapy backbone. 
The observation that the clinical activity of chemoimmunotherapy varies according to disease stage remains unexplained. For example, in stage II and III TNBC, pembrolizumab with chemotherapy improved outcomes across both PD-L1-negative and PD-L1-positive subgroups,${ }^{56}$ whereas in metastatic disease the benefit of pembrolizumab with chemotherapy is restricted to the PD-L1-positive subgroup. ${ }^{12}$ For this reason, one aim of this biomarker study was to characterize the initial state of peripheral blood T-cell populations of mTNBC. T-cell subset quantities and clonal T-cell receptor (TCR) repertoire diversity were assessed in a phase $\mathrm{Ib}$ trial cohort of mTNBC patients, compared with a cohort of earlystage breast cancer (ESBC) patients. A second aim was to assess for changes to T-cell subset quantities and TCR clonal repertoire diversity in patients with $\mathrm{mTNBC}$ after combined pembrolizumab and capecitabine or paclitaxel. Effects of chemoimmunotherapy were compared with those of dose-dense anthracycline and cyclophosphamide (ddAC), which is a commonly used regimen that many patients with $\mathrm{mTNBC}$ receive prior to their diagnosis of metastatic disease; studying its effects on T cells enhances our understanding of the initial T-cell milieu of patients with mTNBC. Importantly, the samples from early-stage patients receiving ddAC were collected contemporaneously using identical biospecimen collection/processing methods and assays.

Measures of T-cell clonal diversity, such as richness (the numbers of unique T-cell clones) and clonality (evenness of clonal abundance within a population), have been correlated with outcomes following immune checkpoint antibody therapy in various cancer types (lung cancer, melanoma, pancreatic cancer, and urothelial cancer),${ }^{7-11}$ as well as following chemotherapy in breast cancer. ${ }^{12-14}$ One explanation for this is that peripheral $\mathrm{T}$ cells are a reservoir of tumor-reactive $\mathrm{T}$ cells,${ }^{15-17}$ thus, the richness or clonality of peripheral $\mathrm{T}$ cells may be useful as a surrogate measure for the ability to mount a successful antitumor response. However, one downside of generalized T-cell diversity metrics is that they cannot distinguish between clonal shifts that are attributable to specific antitumor activity from bystander activation or infection. Simultaneous detection of T-cell clones in both tumor and peripheral blood compartments using TCR immunosequencing has been demonstrated in a variety of cancer types. ${ }^{15}{ }_{18-20}$ Proliferation of tumor-associated T-cell clones in the peripheral blood as a consequence of localized T-cell activation during treatment for mTNBC remains relatively unexplored. Our last aim was to determine whether intratumoral T-cell clones undergo greater proliferation, as measured by abundance, within the peripheral blood following therapy compared with T-cell clones restricted to the blood. If so, this would have represented a novel surrogate biomarker to measure treatmentrelated expansion of putative tumor-reactive T-cell clones. To measure the proliferation rate, we applied regressionbased methods to interrogate the clonal dynamics of T-cell populations.
Monitoring peripheral blood T-cell subsets and T-cell clonal diversity during treatment can elucidate the variable biological effects of various chemoimmunotherapy regimens, particularly in the context of small investigator-initiated studies where samples can be uniformly collected and processed. In previous studies in melanoma, the percentage of individual peripheral T-cell subsets measured by flow cytometry was correlated with clinical response to anti-PD- $1,{ }^{21}$ which could be related to the fact that peripheral $\mathrm{T}$ cells can be an important source of tumor-reactive T cells. ${ }^{16}{ }^{17}$ Additionally, overall lymphocyte count has correlated with clinical benefit from chemotherapy in breast cancer. ${ }^{12}{ }^{14}$ Both paclitaxel and capecitabine are commonly used for the palliative treatment of mTNBC. Their immunological effects have been reported ${ }^{22-27}$ yet their effects when combined with checkpoint blockade have not been examined. We present the first comparison of the effects of pembrolizumab combined with paclitaxel or capecitabine on T-cell subset quantities and T-cell clonal repertoire diversity during first or second-line treatment of patients with mTNBC.

\section{METHODS \\ Participants and sample collection}

Participants with mTNBC $(n=29)$ were enrolled in a phase Ib clinical trial between 2016 and 2018 at Providence Cancer Institute (Portland, OR) and CedarsSinai Medical Center (Los Angeles, California,USA) (NCT02734290). This study evaluated the safety of first or second-line pembrolizumab $200 \mathrm{mg}$ intravenously) on day 1 of a 3-week cycle, combined with either paclitaxel $80 \mathrm{mg} / \mathrm{m}^{2}$ intravenously weekly on days $1,8,15$ of a 3-week cycle $(\mathrm{n}=15)$ or oral capecitabine $2000 \mathrm{mg}$ two times per day on days 1-7 of a 2-week cycle $(n=14)$; chemotherapy was selected by the treating physician. All patients receiving paclitaxel additionally received dexamethasone 10-12 mg intravenously on cycle 1 day 1 . Typically, this was continued weekly until day 15 , when a taper to half the previous dose was attempted with subsequent discontinuation at cycle 2 by the treating clinician's discretion. Only two of nine patients for whom these data are available continued to receive steroids beyond the third week of paclitaxel treatment. Peripheral blood samples were collected at baseline and every 3 weeks for the first 12 weeks of treatment. Tumor samples were collected at baseline and, when possible, a second biopsy was performed between weeks $5-8$. For nine participants, the two biopsies were site matched. This trial met its safety endpoint of at least 6 weeks of treatment without dose discontinuation in $100 \%$ of patients in the capecitabine arm $(14 / 14)$ and $87 \%(13 / 15)$ of patients in the paclitaxel arm. Response outcomes and safety data are reported elsewhere. ${ }^{28}$ Briefly, in the capecitabine arm, overall response rate (ORR) by response evaluation criteria in solid tumors V.1.1 was $43 \%$ (complete response $(\mathrm{CR})=1$, partial response $(\mathrm{PR})=5$, stable disease, $\mathrm{SD}=2$, progressive disease $(\mathrm{PD})=7$ ) while 
Table 1 Demographic data for subjects included in this study

\section{Experimental cohort: metastatic TNBC clinical trial}

\begin{tabular}{|c|c|c|c|}
\hline & No. patients & Average age (median, IQR) & \\
\hline & 29 & $59(61,15)$ & \\
\hline Treatment arm & & 12-week response & No. patients \\
\hline \multirow{2}{*}{ Capecitabine } & & PR & 5 \\
\hline & & SD & 2 \\
\hline Paclitaxel & & SD & 1 \\
\hline
\end{tabular}

\section{Subgroup}

Chemotherapy-naïve 5

$>12$ months since chemotherapy

\section{Comparator cohort: early-stage breast} cancer

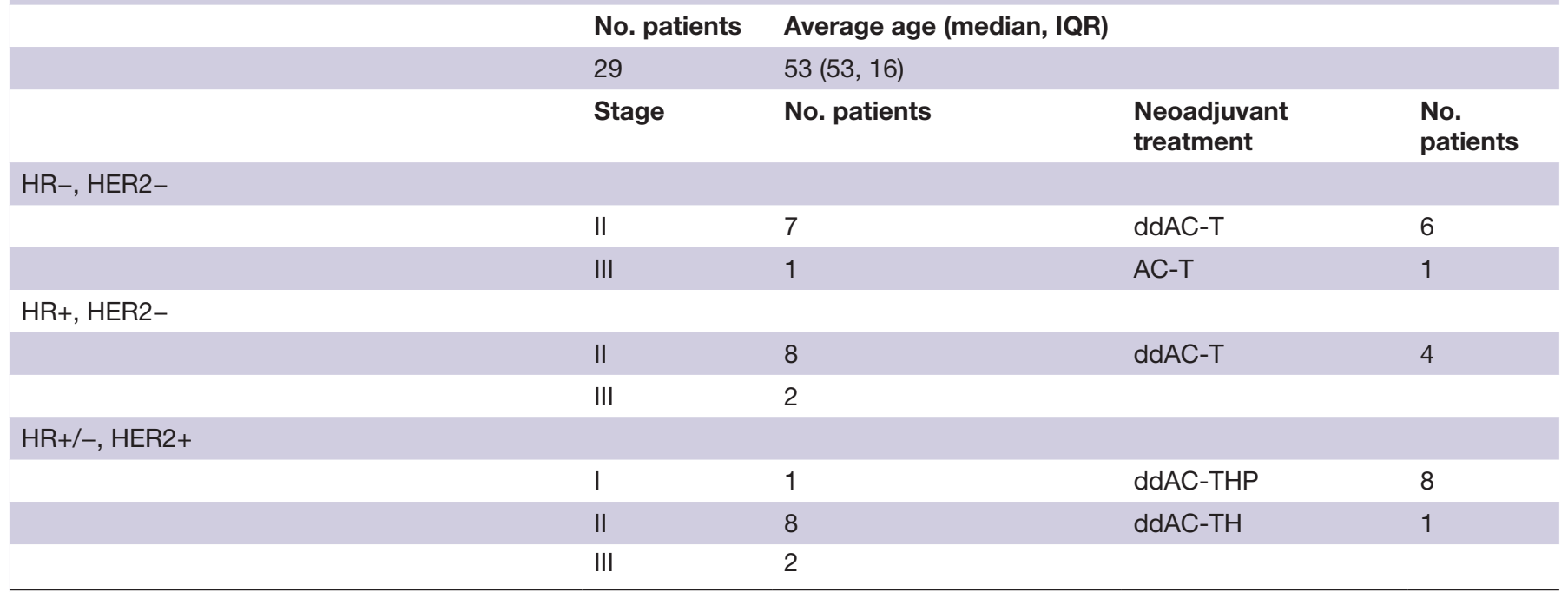

$\mathrm{CR}$, complete response; ddAC, dose-dense anthracycline and cyclophosphamide; $\mathrm{H}$, trastuzumab; HER2, human epidermal growth factor receptor 2; HR, hormone receptor; IQR, interquartile range; P, pertuzumab; PD, progressive disease; PR, partial response; SD, stable disease; T, paclitaxel; TNBC, triple-negative breast cancer.

in the paclitaxel arm, ORR was $29 \%(\mathrm{CR}=2, \mathrm{PR}=2, \mathrm{SD}=1$, $\mathrm{PD}=8$ ) with one patient not evaluable at the cut-off but later assessed as a PR (table 1).

A contemporaneous cohort of patients with ESBC $(n=29)$ was enrolled on a biospecimen collection protocol at Providence Cancer Institute. This presented a unique opportunity to characterize and then compare the immunological effects of curative-intent chemotherapy with chemoimmunotherapy using identical biospecimen protocols and assays. Nineteen subjects received four cycles of doxorubicin $60 \mathrm{mg} / \mathrm{m}^{2}$ intravenously and cyclophosphamide $600 \mathrm{mg} / \mathrm{m}^{2}$ on day 1 of a 14-day cycle, as part of the standard-of-care curative-intent therapy for stage II-III TNBC. To augment the sample size, patients with non-TNBC breast cancer subtypes were included in this analysis if they received the same dose and schedule of ddAC (table 1). Serial blood collections were performed during treatment at baseline and weeks 3, 5 and 9, corresponding to the doxorubicin-cyclophosphamide treatment phase, and when available an additional collection was performed between weeks 18-22.

All patients provided written, informed consent.

\section{Flow cytometry}

Flow cytometry was used to determine $\mathrm{CD} 45^{+} \mathrm{CD} 3^{+} \mathrm{T}$-cell quantities and percentages at the Earle A. Chiles Research Institute (Portland, OR). Fresh whole blood was used to avoid deleterious effects of cryopreservation on cell count estimation. $^{29} 30$ Blood samples collected from patients enrolled at Cedars-Sinai constituted the minority of specimens $(n=8)$ and were collected using the Cyto-Chex BCT tube (Streck La Vista, NE), shipped to the Earle A. Chiles Research Institute, and analyzed within 24 hours of collection. In a separate validation cohort, absolute T-cell counts using this approach were highly correlated with counts obtained from heparinized tubes (data not 
shown). The detailed protocol, including the gating strategy was described previously. ${ }^{31}$ The following $\mathrm{CD}^{+}$ $\mathrm{CD}^{+}$and $\mathrm{CD}^{+} \mathrm{CD}^{+}$T-cell subsets were measured: naïve $\left(\mathrm{CD} 45 \mathrm{RA}^{+} / \mathrm{CCR}^{+}\right)$; central memory (CM) $\left(\mathrm{CD} 45 \mathrm{RA}^{-} /\right.$ CCR7 $7^{+}$); effector memory (EM) $\left(\mathrm{CD}^{2} 5 \mathrm{RA}^{-} / \mathrm{CCR}^{-}\right)$; EM cell re-expressing CD45RA (EMRA) $\left(\mathrm{CD}^{2} 5 \mathrm{RA}^{+} / \mathrm{CCR}^{-}\right.$ ). $\mathrm{CD} 4^{+} \mathrm{T}$ regulatory cells (Tregs) were characterized as $\mathrm{CCR} 4^{+}, \mathrm{CD} 127^{\text {low }}, \mathrm{CD} 25^{\text {high }} .32$

\section{TCR immunosequencing}

For assessment of T-cell clones, DNA was purified from PBMCs using the AllPrep DNA/RNA/miRNA Universal Kit (50) (QIAGEN, Cat \# 80224) with QIAshredder Kit (50) (QIAGEN, Cat \# 79654) for homogenization. DNA was purified from formalin-fixed paraffin-embedded (FFPE) tumor specimens using the AllPrep FFPE Kit (50) (QIAGEN, Cat \# 80234). Purified DNA was submitted for high-throughput deep sequencing using the immunoSEQ TCRB Assay (Adaptive Biotechnologies, Seattle, WA) ${ }^{33-35}$ mTNBC samples collected at baseline and weeks 4,7 and 13 and ESBC samples collected at baseline, weeks 3, 9, and between weeks 18 and 22 were submitted.

\section{Analysis of TCR immunosequencing data}

Various statistical methods were employed to evaluate T-cell clones over time in the peripheral blood and tumor. The T-cell richness, clonality, and differential abundance metrics are commonly cited in the literature ${ }^{36-38}$ and can be directly accessed using the immunoSEQ Analyzer software platform (Adaptive Biotechnologies, Seattle, WA), and are summarized below. We applied regression methods (not yet cited in the literature for this application) to evaluate T-cell clones over time, and to compare effects across treatment groups.

\section{T cell richness}

Clonal richness is one facet of overall diversity of T-cell clones within a population and describes the total number of unique clones in a sample (identified by each clone's CDR3 $\beta$ amino acid sequence). Because cell counts across different blood draws are not standardized, and because the overall sample size can influence the number of unique clones, we present two separate metrics, a unique clone count (which is unadjusted for sample size), as well as T-cell richness, which is adjusted for sample size. T-cell richness is calculated as the average number of unique T-cell clones across 10 random downsampling events (downsampled to 5620 templates for peripheral blood samples, and 248 templates for tumor biopsy samples). ${ }^{36} 39$ The number of templates represent the number of input $\mathrm{T}$ cells after a bias-controlled normalization of TCR immunosequencing reads.

\section{Simpson's clonality}

Clonality is another facet of diversity which describes the evenness of the population. Here, it describes whether $\mathrm{T}$ cells within a population are uniform (all the same clone, monoclonal) or diverse (many different clones, polyclonal). It is calculated as the square root of the
Simpson's diversity index, which incorporates the number of species present and their abundance. High scores indicate an uneven mono/oligo-clonal T-cell population, whereas low scores indicate a more even, polyclonal T-cell population. $^{40}$

\section{Differential abundance}

To assess whether certain T-cell clones are expanded/ contracted in one sample vs another, a statistical test must account for differences related to overall sample size. For this, we utilized Fisher's exact testing with a betabinomial model significance test and multiple hypothesis correction by the Benjamini-Hochberg method. ${ }^{41}$ We also used this method to identify tumor-enriched clones by comparing tumor vs blood samples collected at the baseline timepoint.

\section{Longitudinal analyses}

Serial trends in $\mathrm{T}$ cell subtype or clonal concentrations across multiple time points were analyzed using $\mathrm{R}$ software, V.4.0.3, and the R package 'Ime4'. Various regression methods were employed, as summarized below.

\section{Longitudinal cellular abundance analysis}

To assess the overall rate of $\mathrm{T}$ cell subset expansion or contraction following treatment, we applied a mixed effects longitudinal regression model (with time being a fixed effect, and cell count being the endpoint). This provides an estimate of the rate of change over time (and 95\% CI) for the cellular subset of interest. Variations related to study subject are accounted for by including study subject as a random effect in the model.

\section{Longitudinal $\mathrm{T}$ cell clone detection analysis}

Because chemotherapy is lymphodepleting, we developed linear mixed effects models to quantify relative differences in T-cell clonal loss related to therapy. These models have been used in the longitudinal study of $\mathrm{CD} 4^{+}$ T cell counts ${ }^{42-44}$ and HIV DNA abundance ${ }^{45}$ during antiretroviral treatment for HIV, though to our knowledge have not been applied previously to TCR immunosequencing data. We used a logistic approach to estimate of the odds (ranging from 0 to 1 ) that a $\mathrm{T}$ cell clone would become undetectable during treatment, with values close to 0 indicating that the therapy results in loss of all T-cell clones, and values close to 1 indicating that all T-cell clones remain detectable over time. For the model, $\mathrm{T}$ cell detectability is a binary variable, time is a fixed effect and the subject is a random effect.

A similar strategy was employed to evaluate the OR of the emergence/detection of T-cell clones that were undetectable before starting treatment relative to a reference treatment. The model has a similar structure to the above logistic regression model, but it estimates the odds (ranging from 0 to 1 ) of detection of the subgroup of clones that were not detected in the first sample collection, but were detected later relative to a reference treatment. Values close to 0 would indicate that new T-cell clones are less likely to be detected compared with the 
reference treatment, and values close to 1 would indicate equal likelihood of novel clone detection between the two treatments.

\section{Longitudinal clonotype abundance analysis}

We estimated the mean T-cell clonal proliferation rate related to each treatment using regression models with similar variables as described above. Because there is no reported gold standard method, we explored various regression methods, and found similar outcomes. Details of the various models, including assumptions, merits, and caveats, are summarized in online supplemental table 1. In the results we present outcomes of a Poisson generalized linear mixed model, applied to the subgroups of clones which were enriched within the tumor or were neither enriched within the tumor or not detected in the tumor at all.

We provide a visual method for illustrating the spectrum of T-cell clonal proliferation rates across different subgroups. We calculated the proliferation rate based on the abundance of individual T-cell clones per week, and plotted them in rank order from lowest to highest (by their percentile ranking). To account for possible variations in the rate of clonal proliferation attributable to the starting prevalence, we defined a subgroup of tumorabsent clones with similar baseline frequency to the tumor-present clones. Because these data may be influenced by the overall size of the sample, to compare the subgroups, we randomly sampled 100,000 T-cell clones for each group, with replacement as necessary.

All other statistical analyses to compare T-cell subset quantities and percentages, and diversity metrics were performed using $\mathrm{R}$ software, and included two-sample t-test, Kruskal-Wallis, analysis of variance, and Pearson's linear correlation coefficient testing as indicated. $\mathrm{P}$ values are provided to aid in hypothesis generation but are not corrected for multiple comparisons.

\section{RESULTS}

\section{Baseline T-cell profile of mTNBC patients}

We first characterized baseline peripheral T-cell populations of ESBC and mTNBC patients by flow cytometry and TCR immunosequencing. There were no appreciable differences in T-cell subset sizes within the contemporaneous ESBC cohort according to histology (online supplemental figures 1 and 2). Because there were no differences, data from the entire cohort of ESBC were combined. Patients with mTNBC had fewer lymphocytes than patients with ESBC (CD $45^{+}$ESBC: $2.2 \times 10^{9}$ cells/L, vs mTNBC: $1.2 \times 10^{9}$ cells $/ \mathrm{L}, \mathrm{p}<0.001$ ) (figure $1 \mathrm{~A}$ ). This relation was observed across all T-cell subsets, including $\mathrm{CD}^{+}$cells, $\mathrm{CD}^{+}$cells, $\mathrm{CD} 8^{+}$cells, and naïve, CM, EMRA, and regulatory $\mathrm{T}$ cells (figure $1 \mathrm{~B}-\mathrm{E}$, online supplemental figure 1). figure $1 \mathrm{E}$ lists the average ratio of cell counts across the various T-cell subsets, comparing mTNBC to ESBC. Despite reduced T-cell subset quantities among the mTNBC cohort, the percentages of T-cell subsets relative to all $\mathrm{T}$ cells were similar in the mTNBC vs ESBC cohorts (online supplemental figure 2).

Using TCR immunosequencing, we evaluated T-cell clonal diversity in the mTNBC vs ESBC cohorts. The two cohorts exhibited similar mean T-cell richness (ESBC: 4626 unique rearrangements per 5260 TCR molecules, mTNBC: 4440 unique rearrangements per 5260 TCR molecules, $\mathrm{p}=0.40$, t-test) and clonality (ESBC: 0.055, mTNBC: $0.055, \mathrm{p}=0.98$, t-test) (figure 2A,B). There were no differences in T-cell richness or clonality between the histological subgroups among the ESBC cohort (online supplemental figure 3). We evaluated the relative distribution of T-cell clones according to their abundance and found similar proportions of high-frequency clones between the cohorts, but differences in lower and moderate-frequency clones (figure 2E).

Because chemotherapy can be associated with longterm alterations to peripheral T-cell profiles, ${ }^{46-48}$ we compared different subgroups of mTNBC participants: chemotherapy-naïve $(n=5)$, most recent chemotherapy $>12$ months ago $(\mathrm{n}=16)$, and most recent chemotherapy 6-12 months ago $(n=8)$. The average baseline $\mathrm{CD}^{+}{ }^{+}$T-cell count did not vary significantly among these groups, except for $\mathrm{CD} 8^{+} \mathrm{EM}$ cells (online supplemental figures 1 and 2). Comparisons of richness and clonality were limited due to lower numbers of chemotherapy-naïve patients with available TCR immunosequencing data (data not shown).

\section{Effects of chemoimmunotherapy on peripheral $\mathrm{T}$ cell subsets and TCR repertoire in $\mathrm{mTNBC}$}

We observed similar declines in $\mathrm{CD}^{+} \mathrm{T}$ cells over time whether patients received pembrolizumab with capecitabine or paclitaxel (average $\mathrm{CD}^{+} \mathrm{T}$-cell fold change capecitabine: -0.42 , paclitaxel: $-0.56, \mathrm{p}=0.80$, t-test). By comparison, patients in the ESBC cohort treated with ddAC experienced a greater loss of $\mathrm{CD}^{+} \mathrm{T}$ cells (average fold change: -1.21). Similarly, the rate of $\mathrm{T}$ cell loss across all major T-cell subsets, including Tregs, was greater after ddAC than either of the pembrolizumab-based chemotherapy regimens (figure 3 , online supplemental table 2).

There were no significant changes to TCR repertoire richness or clonality with either capecitabine or paclitaxel combined with pembrolizumab, whereas ddAC resulted in reduced $T$ cell richness but no change in clonality (figure $4 \mathrm{~A}, \mathrm{~B}$ ). There were not differences in the number of significantly expanding (figure 4C) or contracting clones (figure 4D) between patients treated with pembrolizumab combined with capecitabine or paclitaxel. Following ddAC there were more expanded clones and fewer contracted clones than observed after either pembrolizumab-based regimen. However, this finding did not reach statistical significance (figure $4 \mathrm{C}, \mathrm{D}$ ). The expanding clones identified during each treatment were durable and detectable at later time periods (capecitabine: $85 \%$, paclitaxel: $83 \%$; ddAC: $80 \%$ ). In summary, using these generalized metrics, we found minimal differences in T-cell clonality or diversity between the two 
A

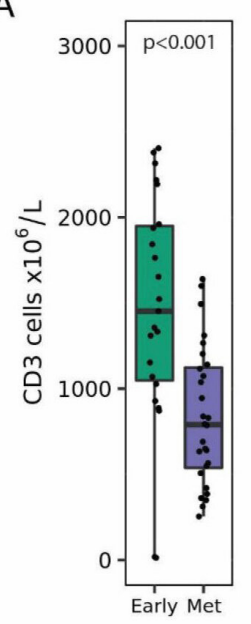

B

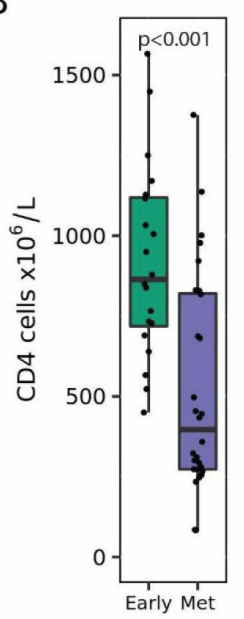

C

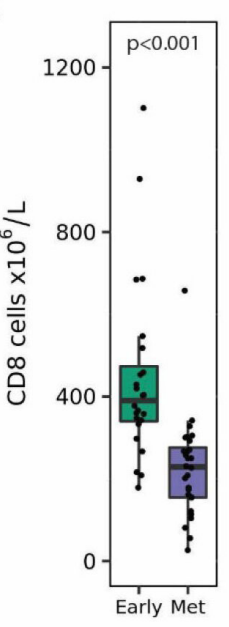

D

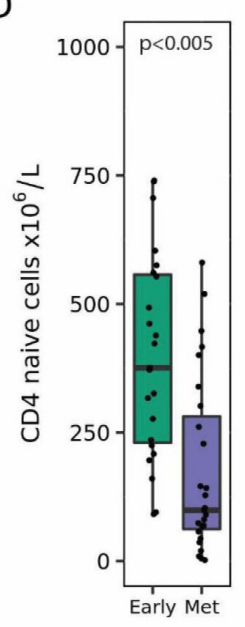

E

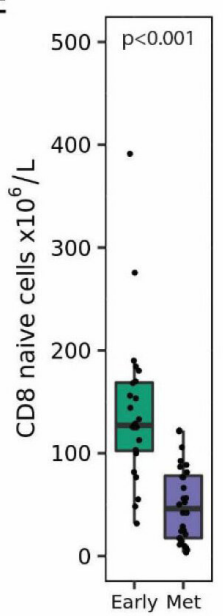

F

\begin{tabular}{|l|r|}
\hline Cell Type & $\begin{array}{l}\text { Ratio of average cell counts, } \\
\text { metastatic: early }\end{array}$ \\
\hline CD45 & 0.56 \\
\hline CD3 & 0.54 \\
\hline CD4 & 0.48 \\
\hline CD8 & 0.50 \\
\hline T reg & 0.67 \\
\hline CD4 naïve & 0.48 \\
\hline CD4 CM & 0.50 \\
\hline CD4 EM & 0.45 \\
\hline CD4 EMRA & 0.38 \\
\hline CD8 naïve & 0.36 \\
\hline CD8 CM & 0.64 \\
\hline CD8 EM & 0.80 \\
\hline CD8 EMRA & 0.38 \\
\hline
\end{tabular}

Figure 1 (A-E) Baseline T-cell subset counts for early-stage breast cancer and mTNBC study cohorts. Additional subset counts and percentages are available in online supplemental figure 1 and 2. Significance testing is by the two-sample t-test. (F) Ratio of the average T-cell subset counts in the metastatic versus early-stage cohorts. CM, central memory; EM, effector memory; EMRA, effector memory re-expressing CD45RA; Met, metastatic; mTNBC, metastatic triple-negative breast cancer; Treg, regulatory $T$ cell.

pembrolizumab-based regimens, whereas the curativeintent ddAC regimen was associated with a loss of overall diversity and evidence of more T-cell clonal expansions.

We applied regression methods to interrogate further the effect of these therapies on the T-cell clonal repertoire. Regarding T-cell clones, we confirmed that the rate of clonal loss between the pembrolizumab-based arms were similar, and lower than observed after ddAC (figure 4E). On the other hand, pembrolizumab with chemotherapy resulted in fewer T-cell clones emerging during treatment than arose during ddAC (figure 4E). In summary, these data may suggest that ddAC administered for early-stage treatment facilitates robust peripheral T-cell clonal proliferation despite great lymphotoxicity.

\section{Changes to intratumoral T cells during chemoimmunotherapy for mTNBC}

From the mTNBC patients, we obtained nine pairs of pretreatment and on-treatment tumor samples from the same lesion, providing an opportunity to evaluate changes to intratumoral lymphocytes during treatment.
Overall, changes in intratumoral T-cell richness and clonality varied across patients and no specific trend could be detected (figure 5A,B). However, pretreatment/ post-treatment comparisons were not evaluable for all pairs because of low numbers of TCR templates in some samples. The number of $\mathrm{T}$ cells detected by TCR immunosequencing increased in some pairings, but not in all (figure 5C); however, the fraction of $\mathrm{T}$ cells among all nucleated cells increased in most samples (figure 5D).

On average, each patient had 771 (range 1-4964) T-cell clones that were present in both baseline and on-treatment biopsies. On average, these clones comprised 19\% of the baseline clones (range: $0.1 \%-36 \%$ ) and $17 \%$ of the on-treatment clones (range: $3 \%-41 \%$ ). In five subjects we identified T-cell clones that significantly expanded or contracted following therapy at an alpha level of 0.05 (figure 5F). Expanding T-cell clones were more likely to be detected in the peripheral blood, when compared with those that contracted (expanding: 80\%; contracting: $43 \%$ ). However, expansion or contraction of $\mathrm{T}$ cell clones 
A

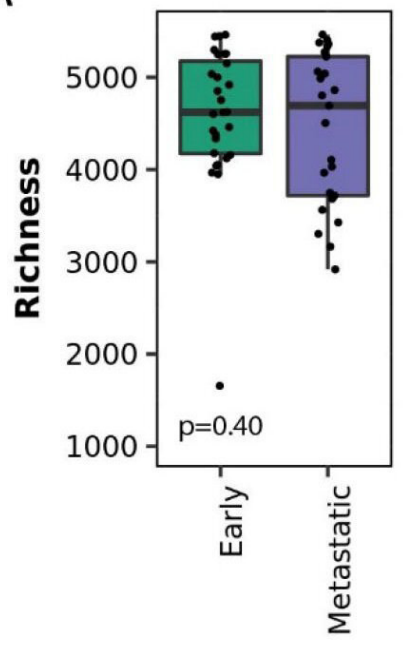

B

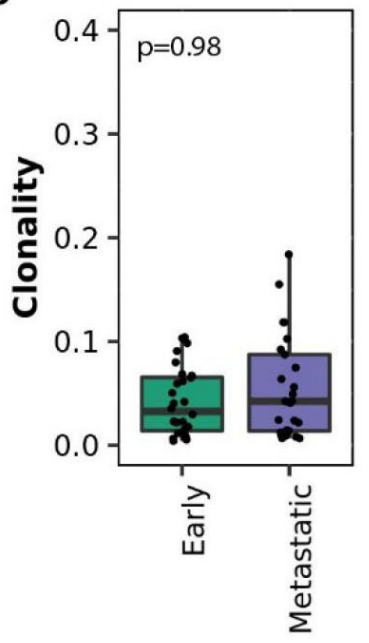

C

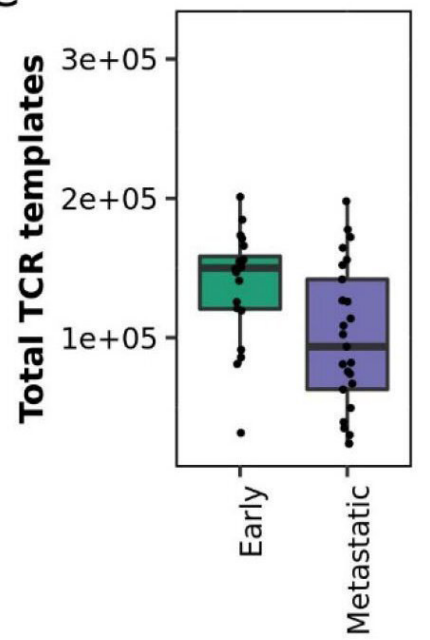

D

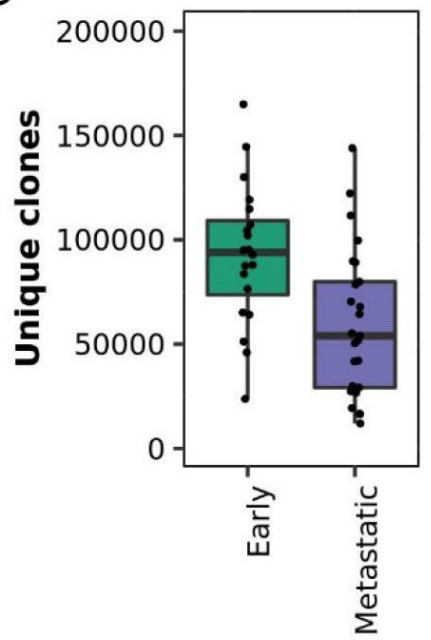

$\mathrm{E}$

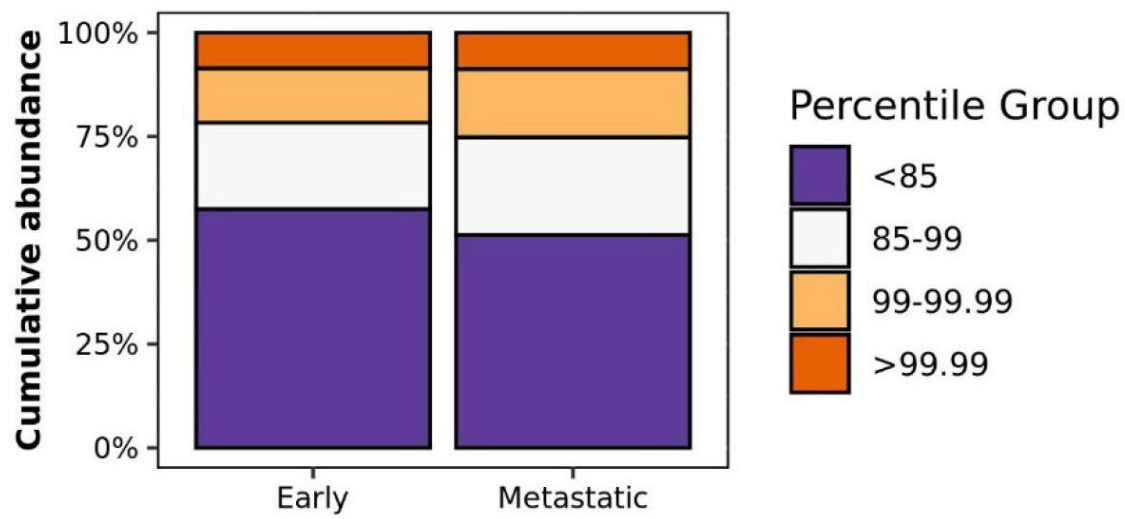

Figure 2 Baseline TCR repertoire diversity metrics for early-stage breast cancer and metastatic triple-negative breast cancer cohorts as measured by $(A)$ richness, $(B)$ clonality, $(C)$ total TCR templates per sample and (D) total unique clones. Significance testing is by the two-sample t-test. (E) Average cumulative abundance of clones grouped by prevalence. TCR, T-cell receptor.

in the tumor did not always correlate with expansion or contraction in the peripheral blood (figure 5E). Out of the three patients with expansion of at least one clone in both the tumor and the blood, only one patient had a PFS greater than the average for the entire cohort of mTNBC patients (patient PFS: 258 days; cohort mean PFS: 146.3 days, range: $21-426$ days) (figure $5 \mathrm{E}$ ). TCR CDR3 $\beta$ amino acid sequences of differentially-abundant clones within tumors (online supplemental table 3 ) did not match any previously-identified sequences within publicly available $\mathrm{V}(\mathrm{D}) \mathrm{J}$ databases McPAS-TCR ${ }^{49}$ and VDJ-db ${ }^{50}$ though the amount of available data is currently limited.

\section{Use of tumor specimens to identify and track tumor-enriched TCR clones}

We explored whether longitudinal trends of peripheral T-cell clone abundance differed based on the clone's presence within pretreatment tumor. Pretreatment tumor and serial blood samples were available from 19 mTNBC clinical trial patients which facilitated the identification of intratumoral T-cell clones, some of which are putatively tumor-reactive. Interestingly, there were seven clones which were expressed in both tumor and blood compartments, and detected in more than one person. Expression of a shared clone was not necessarily associated with clinical response (online supplemental table 4). Among clones present within the tumor, their abundance was loosely correlated to their average relative abundance in the blood (figure 6A), raising the alternate hypothesis that T-cell clones in the tumor are related to tissue perfusion by peripheral T-cell clones, which are not necessarily tumor-reactive.

To explore these contrasting possibilities, we looked for differences in trends of clonal abundance among a subset of intratumoral T-cell clones that were enriched within the tumor, relative to the peripheral blood (using the differential abundance statistical test). We identified 1914 tumor-enriched clones among the 19 patients (average per patient: 101, range: 0-469) out of 145,534 tumor-present T-cell clones (average per patient: 7660, range: 79-54,714) (online supplemental figure 4B). On average, tumor-enriched clones comprised $0.79 \%$ of the total detected TCR sequences within the peripheral blood compartment and $20.2 \%$ within the tumor compartment (online supplemental figure 4B). Tumor-present 

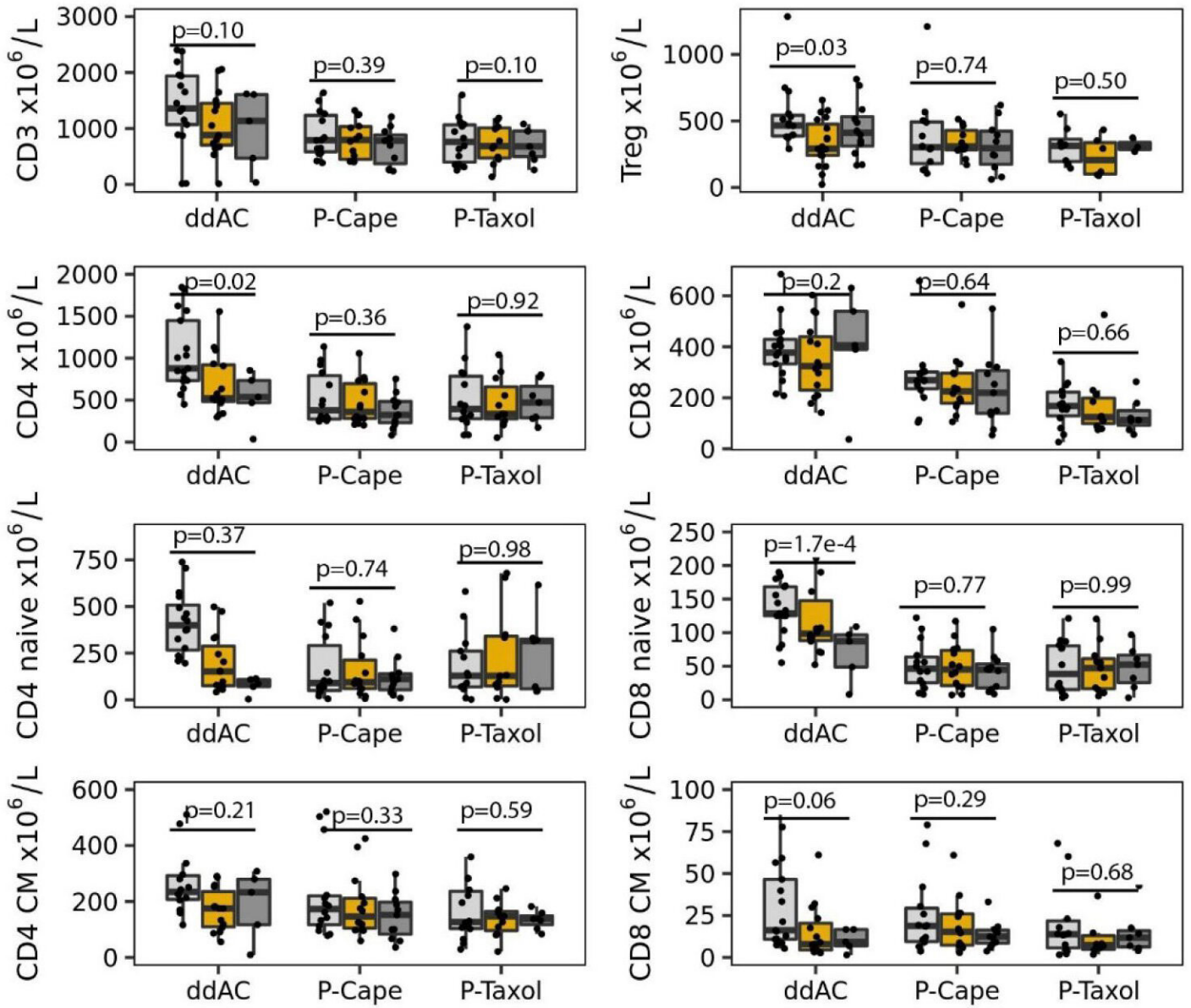

Time point
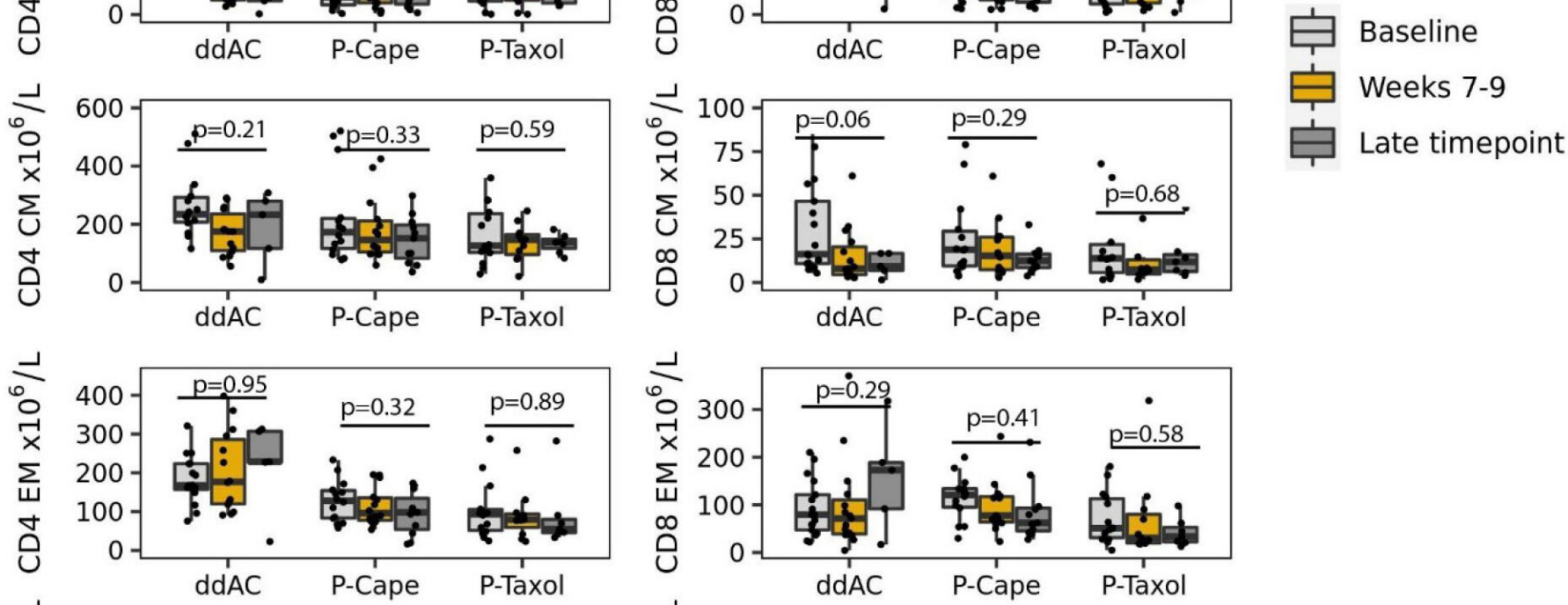

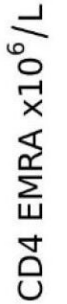
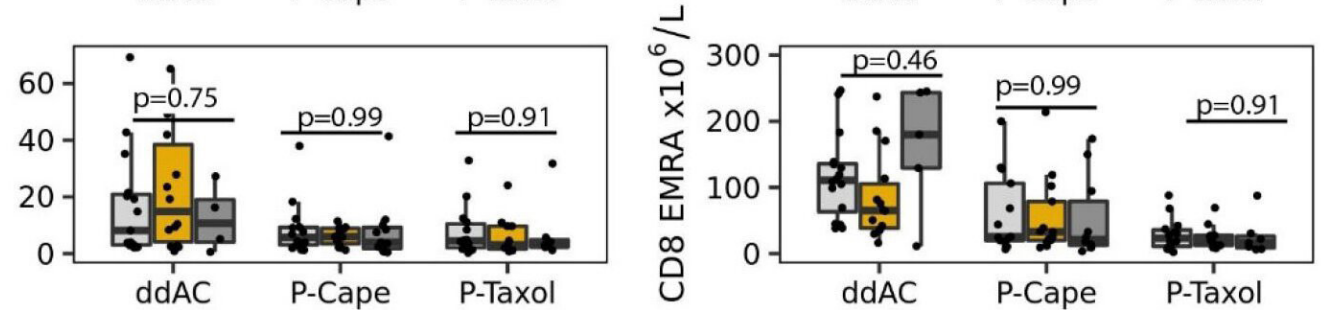

Figure 3 T cell subset counts during treatment with ddAC for early-stage patients and pembrolizumab and capecitabine or paclitaxel for mTNBC. The late timepoint was week 13 for P-Cape and P-Taxol and between weeks 19-22 for ddAC. Significance testing is by ANOVA. ANOVA, analysis of variance; ddAC, dose-dense doxorubicin, cyclophosphamide; CM, central memory; EM, effector memory; EMRA, effector memory re-expressing CD45RA; mTNBC, metastatic triple-negative breast cancer; P-Cape, pembrolizumab and capecitabine; P-Taxol, pembrolizumab and paclitaxel; Treg, regulatory T cell.

clones comprizing the remaining $79.8 \%$ of tumor TCR sequences comprised $18.7 \%$ of TCR sequences within the peripheral blood. There were no tumor-enriched clones from baseline samples which were shared between subjects.

Tumor-present and tumor-enriched clones were more abundant in the blood on average than tumor-null clones (average TCR molecules per clone at week 7, tumor-enriched: 8.8, tumor-present: 1.8, tumor-absent: 0.39 ), but the average rate of change in the blood was similar across these three groups (figure $6 \mathrm{~B}$, online supplemental table 5). The quantile plot (figure 6C) illustrates the individual slopes of clone abundances (rate of change) identified in the groups: tumor-enriched, tumor-nonenriched, and peripheral blood matched and unmatched for starting abundance. As illustrated, the 
A

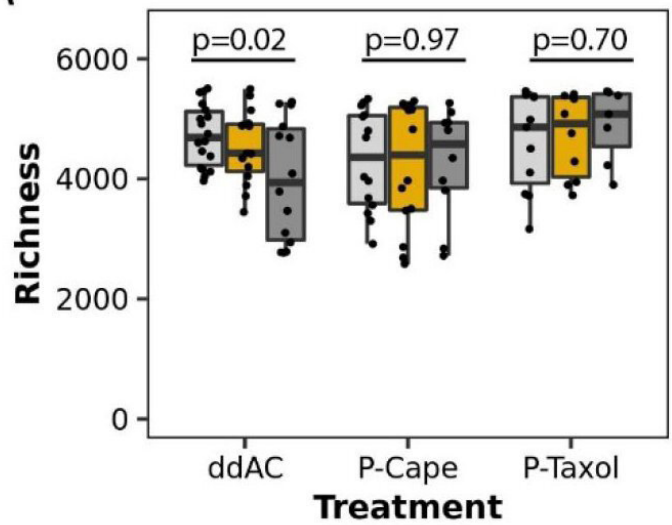

C

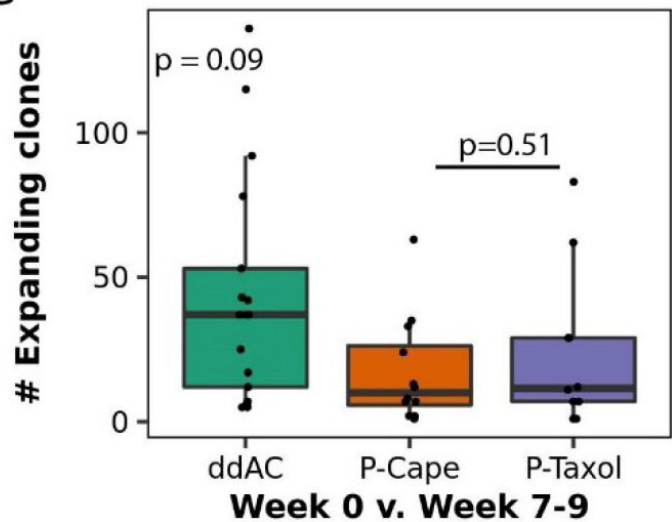

B

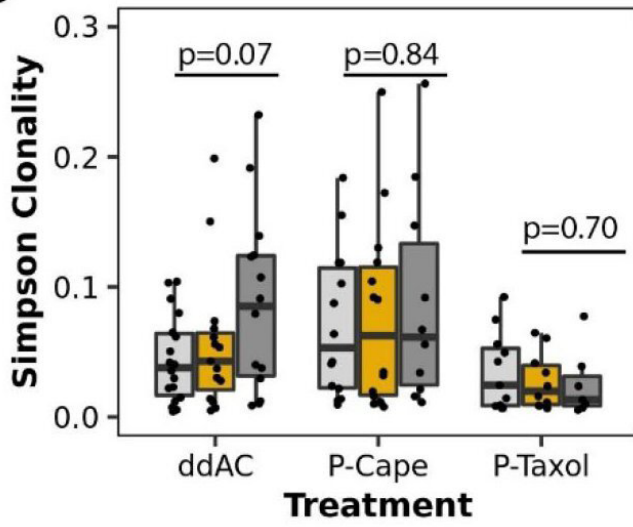

Time point

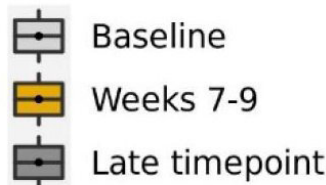

D

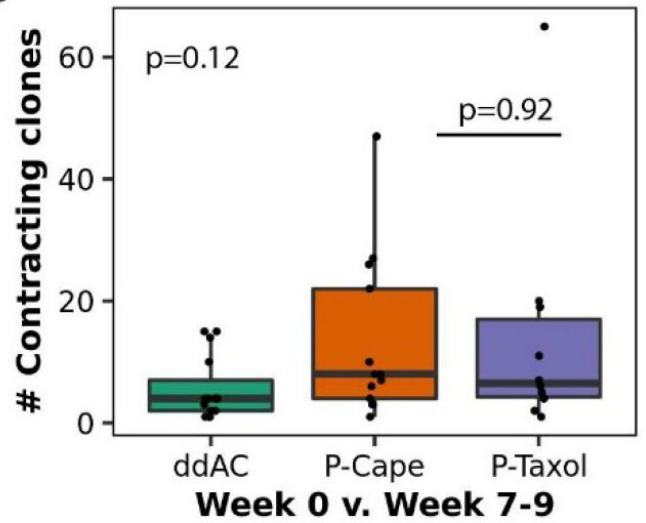

$\mathrm{E}$

\begin{tabular}{|c|c|c|}
\hline \multicolumn{3}{|l|}{ Weekly loss of clones } \\
\hline Treatment group & Odds Ratio & $95 \% \mathrm{Cl}$ \\
\hline$d d A C$ & 0.899 & $0.898-0.899$ \\
\hline P-Cape & 0.957 & $0.957-0.958$ \\
\hline P-Taxol & 1.017 & $1.016-1.018$ \\
\hline \multicolumn{3}{|c|}{ New clone detection during treatment } \\
\hline Treatment group & Odds Ratio & $95 \% \mathrm{Cl}$ \\
\hline P-Cape (reference ddAC) & 0.292 & $0.286-0.299$ \\
\hline P-Taxol (reference ddAC) & 0.652 & $0.625-0.681$ \\
\hline P-Cape (reference P-Taxol) & 0.455 & $0.434-0.477$ \\
\hline
\end{tabular}

Figure 4 TCR diversity $(A, B)$ and numbers of differentially abundant clones per patient $(C, D)$ during treatment. The late time point was week 13 for P-Cape and P-Taxol and between weeks 19-22 for ddAC. Significance testing is by the ANOVA test (A, B). The t-test was used for P-cape vs P-taxol, Kruskal-Wallis testing was used for three-way treatment comparison (C, D). (E) The odds of losing clones per week (top) and detecting novel clones per treatment (bottom). ANOVA, analysis of variance; ddAC, dose-dense anthracycline and cyclophosphamide; P-Cape, pembrolizumab with capecitabine; P-taxol, pembrolizumab with paclitaxel.

abundance in the blood of most T-cell clones is stable over time across groups, and rare clones expand or contract.

\section{DISCUSSION}

In this post hoc analysis of potential biomarkers, we employed comprehensive T-cell monitoring using fresh whole-blood flow cytometry and blood/tissue TCR immunosequencing of blood and tissue to provide insights into the starting T-cell profile of patients with mTNBC, and the subsequent effects of palliative chemoimmunotherapy on T-cell populations. We examined alterations in T-cell populations during ddAC treatment in an earlystage cohort as a comparator to aid hypothesis generation regarding the development of immunosuppression which may be prevalent in mTNBC patients. We addressed three aims: (1) to characterize baseline T-cell profiles of 
A

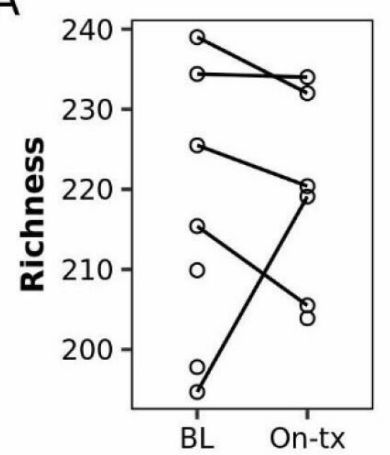

B

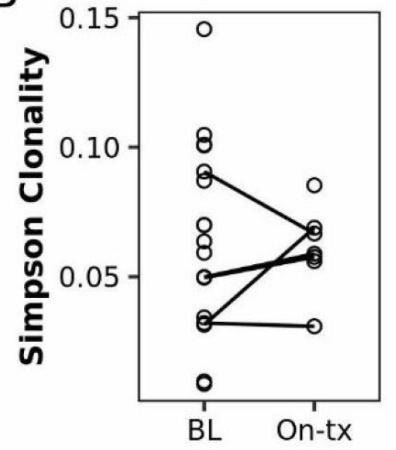

C

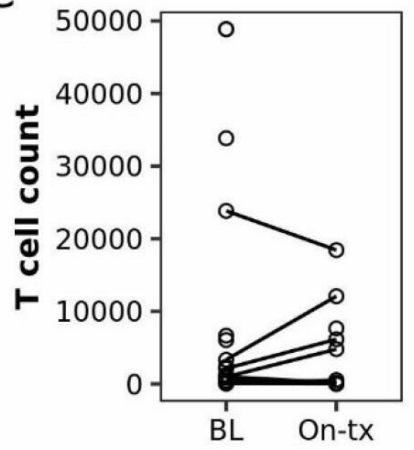

D

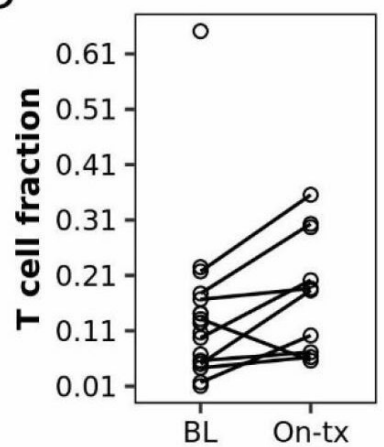

E

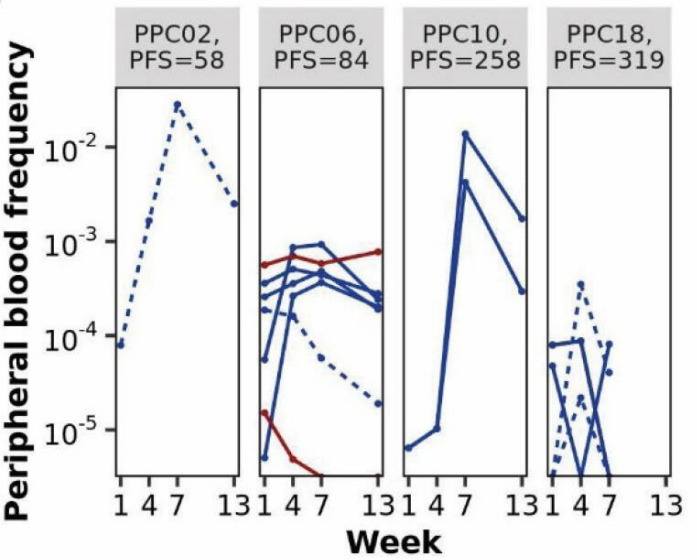

Significance level

— alpha $=0.05$

.... alpha $=0.10$

Group

- Contracting

- Expanding

$\mathrm{F}$

\begin{tabular}{|l|r|r|r|r|r|r|r|r|}
\hline Subject & $\begin{array}{l}\text { Fold change } \\
\text { clone count }\end{array}$ & $\begin{array}{l}\text { Fold change } \\
\text { template } \\
\text { count }\end{array}$ & $\begin{array}{l}\text { Fold change } \\
\text { clonality }\end{array}$ & $\begin{array}{l}\text { Fold change } \\
\text { richness }\end{array}$ & $\begin{array}{l}\text { Morisita } \\
\text { Index }\end{array}$ & $\begin{array}{l}\text { \# clones } \\
\text { expanding }\end{array}$ & $\begin{array}{l}\text { \# clones } \\
\text { contracting }\end{array}$ & $\begin{array}{l}\text { 12-week } \\
\text { response }\end{array}$ \\
\hline PPC06 & 2.07 & 2.60 & 1.16 & -1.05 & 0.77 & 4 & 4 & PD \\
arm
\end{tabular}

Figure 5 (A-D) comparison of pretreatment and on-treatment TIL TCR immunosequencing data (E) Peripheral blood abundances of tumor differentially abundant clones. (F) Summary table of five paired tumor samples adequate for diversity assessment, numbers of expanding and contracting clones were calculated with alpha level 0.05 . PPC27 did not have paired peripheral blood immunosequencing data. BL, baseline; On-tx, on-treatment; PFS, progression-free survival; PD, progressive disease; PR, partial response; SD, stable disease; TCR, T-cell receptor.

patients with $\mathrm{mTNBC}$ by $\mathrm{T}$ cell subset quantity and clonal repertoire; (2) to characterize the effects of two different chemoimmunotherapy regimens on T-cell subsets and clonal repertoire; and (3) to explore whether the peripheral proliferation rate of clones that are enriched within the tumor is different than that of non-enriched clones.

We characterized the baseline peripheral blood T-cell counts and clonal diversity in patients with $\mathrm{mTNBC}$ relative to ESBC because baseline differences in the T-cell profile may contribute to observed differences in clinical efficacy of chemoimmunotherapy between early and metastatic stage TNBC. In our analysis, mTNBC patients exhibited global reductions in peripheral T-cell quantities relative to ESBC counterparts, with absolute cell counts of T-cell subsets in the metastatic setting ranging from $36 \%$ to $80 \%$ of counts observed in ESBC. Furthermore, while mean T-cell clonal diversity scores were similar in mTNBC and ESBC, there was greater variability in these metrics within the mTNBC cohort, highlighting the heterogeneity of patients within the metastatic cohort. 
A

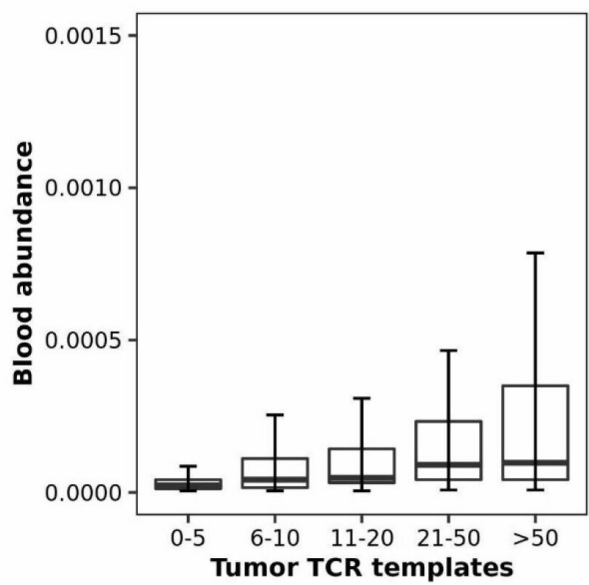

B

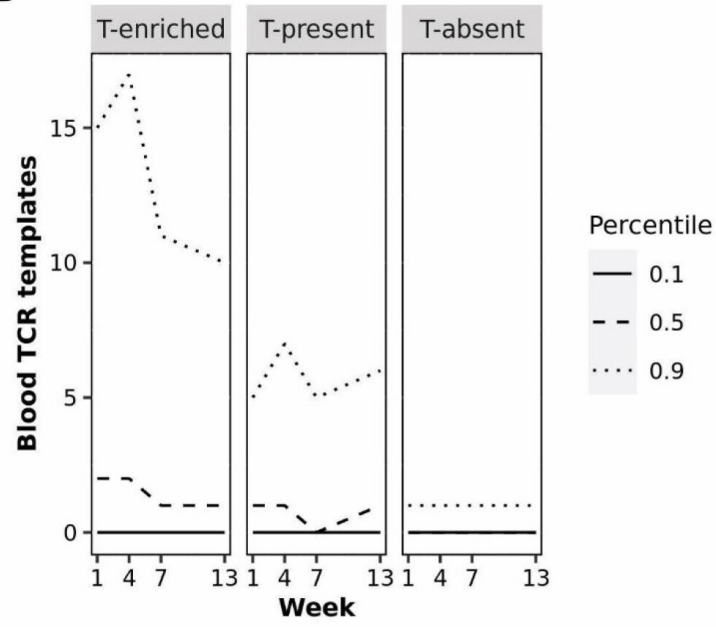

$\mathrm{C}$

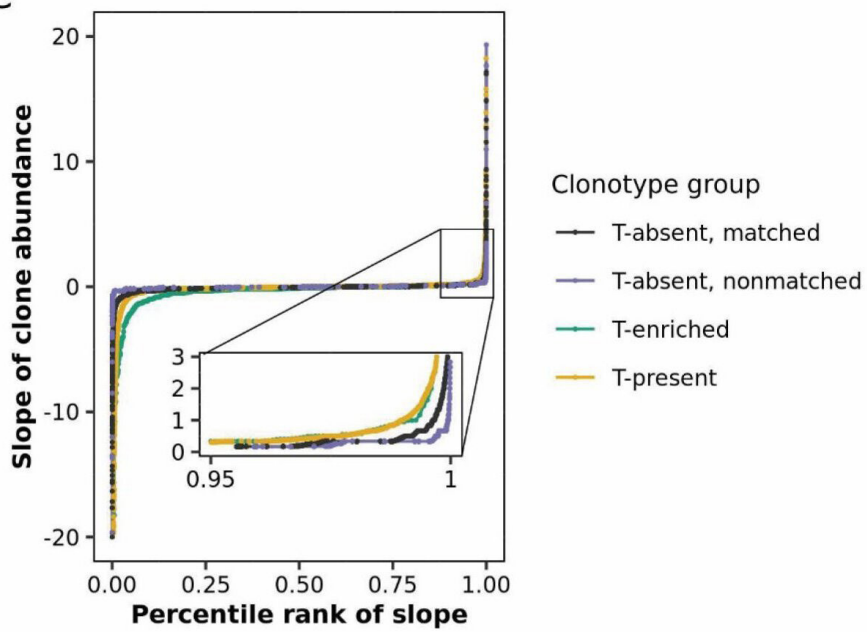

Figure 6 (A) Clone abundances within the blood versus tumor (B) TCR reads within the blood, per clone over time. (C) Illustration of the distribution of weekly slopes of clone abundance, per group. Slopes were calculated as the rate of change in TCR templates per week and are arranged in order of percentile rank. A total of 100,000 clones were sampled randomly from each group. TCR, T-cell receptor; T-enriched, tumor-enriched; T-present, tumor-present; T-T-absent, tumor-absent.

We also detected differences in the cumulative proportion of low/moderate-abundance T-cell clones in the overall TCR repertoire. Overall, these data suggest that the systemic T-cell milieu is suppressed in patients with mTNBC. Whether this directly relates to differences in observed efficacy of checkpoint inhibition in metastatic and early-stage TNBC is worthy of further study.

Important questions remain regarding whether our observation of relative lymphodepletion in patients with mTNBC is a consequence of previous therapies resulting in bone marrow suppression, and whether $\mathrm{T}$ cells in patients previously treated with chemotherapy have reduced functional capabilities. Although the dataset is too small to provide definitive conclusions, we observed that the timing of recent curative-intent therapy in mTNBC patients did not impact $\mathrm{T}$ cell concentrations or richness. There were some numerical differences between the chemotherapy-naive patients, and those previously treated with chemotherapy, which is supported by literature that curative-intent chemotherapy results in long-term $\mathrm{T}$ cell depletion, particularly of $\mathrm{CD} 4^{+}$subsets. ${ }^{46-48}$ Understanding the long-term effects of previous therapies on T-cells is worthwhile as chemoimmunotherapy appeared to confer greater benefit in PFS for chemotherapy-naïve patients in the IMpassion130 and Keynote-355 trials. ${ }^{12}$ Additionally, in a previous phase II study, pembrolizumab failed to improve PFS when combined with capecitabine in later lines of therapy, ${ }^{4}$ which contrasts with our phase Ib trial that reported a more favorable PFS of pembrolizumab plus capecitabine when administered earlier in the disease course. ${ }^{28}$ Other factors, such as tumor burden ${ }^{51}$ and age $\mathrm{e}^{52-54}$ may contribute to immunosuppression and may be relevant in patients with mTNBC.

We characterized the effect of pembrolizumab plus capecitabine or paclitaxel on T-cell subsets and the TCR clonal repertoire. Both chemotherapies are used 
commonly in the palliative treatment of mTNBC and are safe in combination with pembrolizumab. ${ }^{2428}$ While immune effects of these chemotherapies as single agents have been described, the effect of adding pembrolizumab has not been examined. The effects could be beneficial or harmful. Capecitabine and its active moiety, 5-fluorouracil, may enhance peptide presentation by MHC-I in tumor cells ${ }^{55}$ deplete myeloid-derived suppressor cells (MDSCs), ${ }^{23}$ and increase T-helper 17 cells $\left(\mathrm{T}_{\mathrm{H}} 17\right.$ cells $) .{ }^{22}$ Paclitaxel repolarizes macrophages to an antitumor M1 phenotype, ${ }^{24}$ depletes MDSCs, ${ }^{25}$ and promotes dendritic cell maturation and function. ${ }^{26}$ There is additional concern that corticosteroid prophylaxis, routine with paclitaxel therapy, but not capecitabine, may contribute to suppressed $\mathrm{T}$ cell viability, signaling, and function. ${ }^{567}$ When capecitabine and paclitaxel were combined with pembrolizumab, we did not observe meaningful differences in T-cell subset losses using conventional surface markers nor in T-cell clonal expansion using previously established methods of assessing T-cell clonal diversity or differential abundance. Using a novel application of logistic regression, emergence of novel clones was more likely to occur following paclitaxel than capecitabine chemoimmunotherapy. Emergence of new clones was even more likely during ddAC. This finding requires additional confirmatory study, but generates interest because novel clones which emerge during treatment may have the potential to target tumor-associated neoantigens. It is possible that ddAC stimulated the expansion of more clones due to doxorubicin's immunogenic effects, ${ }^{58}$ or that the more severe lymphopenia induced by ddAC triggered rapid endogenous proliferation of T cells. ${ }^{59}$ Additional evaluations to determine any differences between chemotherapy backbones on immune cell populations could include the quantification of additional immune cell subsets, characterization of T-cell function using direct T-cell functional assays or single-cell RNA sequencing or proteomic assays.

The third goal was to characterize dynamics of intratumoral versus peripheral T-cell clonal expansion, in hopes that we could identify a peripheral blood surrogate biomarker for tumor-specific T-cell response related to chemoimmunotherapy. TCR sequencing of tumor digests did not reveal a pattern of changes in intratumoral T-cell clonal diversity related to treatment; however, it identified statistically significant clonal T-cell expansion events in site-matched specimens. When tracked individually, T-cell clones enriched within the tumor (including those found to be expanding) were often detected within the blood, but did not necessarily expand within the blood. Among each population of T-cell clones there is a range of proliferative rates within the blood, with the vast majority of clones undergoing no proliferation and rare outliers either proliferating or contracting. In summary, these data suggest that expansions of intratumoral T-cell clones are detectable within the blood, but these are rare even when focusing on T-cell clones enriched within the tumor, and clones that expand within the tumor can easily be overlooked if one relies solely on the blood to identify potentially tumor-reactive T-cell clones. In future experiments, TCR sequencing of T-cell subsets that are more likely to be tumor-reactive (such as CD103/CD39 double-positive T cells ${ }^{19}$ or T cells with CXCL13, CD39, TOX, and PD-1 expression ${ }^{60}$ could more accurately identify tumor-reactive T-cell clones.

There are limitations to this biomarker analysis. First, as a biomarker-focused investigator-initiated trial, the sample size is small relative to phase III trials such as Keynote-355 and Impassion 130, and therefore, the findings are exploratory and not powered to detect small differences across the treatment arms. However, one virtue of this analysis was that the specimens were processed and analyzed in one laboratory using a standardized protocol and non-cryopreserved blood. This type of analysis is often impossible to conduct using specimens from large, multiinstitutional clinical trials. Limitations regarding the comparison of treatment effects arise because patients were not randomized between capecitabine and paclitaxel, and the contemporaneous ESBC cohort comprised a variety of tumor histotypes receiving ddAC without the inclusion of pembrolizumab. Therefore, it is possible that some of the differences attributed to ddAC are in fact related to the omission of pembrolizumab, differences in tumor subtype, or the immune contexture of early-stage disease. Therefore, it is important that these findings are confirmed in a more homogenous dataset. We will later report immunological profiles of a larger cohort of $n=30$ stage II/III TNBC patients receiving ddAC with pembrolizumab in an ongoing phase II investigator-initiated trial (NCT04373031). Meanwhile, our reported data offers useful insights for other investigators planning to study changes to T-cell populations during immunotherapy trials. A third limitation is that our analysis was restricted to whole-blood flow cytometry and whole-tumor TCR sequencing. For future studies, we advocate for singlecell multiomic characterizations of T-cells to capture TCR clonotype and functional state to better characterize $\mathrm{T}$ cell activity and clonal proliferation in response to treatment.

\section{CONCLUSION}

We present longitudinal immunological profiling of the effect of pembrolizumab with capecitabine or paclitaxel in mTNBC. At baseline, patients with mTNBC, the majority of whom were pretreated, exhibited depletion of T-cell subsets and reduced T-cell clonal richness compared with untreated ESBC patients, but percentages of T-cell subsets and clonality were not significantly different between cohorts. We did not detect significant differences in T-cell subset losses when comparing patients treated with either of the two chemoimmunotherapy arms. Exploratory application of logistic regression techniques revealed differences in the likelihood of emergence of novel clones between the various treatments. Changes to TIL populations were heterogeneous within nine paired tissue samples. Lastly, regression-based 
statistical methods can characterize the peripheral proliferation rate of T-cell clones and are applicable in immune monitoring studies.

\section{Author affiliations}

${ }^{1}$ Earle A Chiles Research Institute, Providence Cancer Institute, Portland, Oregon, USA

${ }^{2}$ Knight Cancer Institute, Oregon Health \& Science University, Portland, Oregon, USA ${ }^{3}$ Medical Data Research Center, Providence St Joseph Health, Portland, Oregon, USA ${ }^{4}$ Computational Immuno-Oncology and Bioinformatics Core, Earle A. Chiles Research Institute, Portland, Oregon, USA

${ }^{5}$ Adaptive Biotechnologies Corp, Seattle, Washington, USA

${ }^{6}$ Department of Medicine, University of Texas Southwestern Medical Center, Dallas, Texas, USA

Twitter William L Redmond @WWredmond4, Walter J Urba @WalterUrba and Heather L McArthur @hmcarthur

Acknowledgements The authors would like to acknowledge collaborators at the Earle A. Chiles Research Institute and Adaptive Biotechnologies for mentorship and guidance. Support for the clinical trial (NCT02734290), which comprised the metastatic cohort was provided by Merck and the Providence Opportunity Fund.

Contributors BC: primary data analysis, manuscript writing. JP: clinical data collection, analysis. SCC: data analysis. IK: clinical data collection. BN: manuscript editing. YK: T cell profiling. WLR: T cell profiling. VR: data analysis. BB: data analysis. VC: data collection. MS: specimen collection. NP: TCR immunosequencing interpretation guidance. PAF: TCR immunosequencing interpretation guidance. WJU: critical review of manuscript. AKC: treatment of study subjects. HLM: treatment of study subjects. DBP: treatment of study subjects, data analysis, study design and conduct, critical review of manuscript, guarantor of content.

Funding Laboratory services were provided at no cost by Adaptive Biotechnologies. Funding was also provided by the Providence Portland Medical Foundation.

Competing interests YK: Research support from Bristol Myers Squibb (BMS), GlaxoSmithKline, Shimadzu. WLR: Research support from Galectin Therapeutics, BMS, GlaxoSmithKline, Mi. NA Therapeutics, Inhibrx, Veana Therapeutics, Shimadzu, OncoSec Medical, Turn Biotechnologies, CanWell Pharma, Aeglea Biotherapeutics, and Calibr. Patents/Royalties: Galectin Therapeutics. Advisory Boards: Nektar Therapeutics, Vesselon. NP: Financial interest: Adaptive Biotechnologies. PAF: Financial interest: Adaptive Biotechnologies. WJU: Safety advisory board: Astra Zeneca. HLM: Research support: Merck, Lilly, BMS. Advisory/ consultancy Merck, Lilly, Spectrum Pharmaceuticals, Amgen, Immunomedics, Pfizer, Genentech, BMS, Genomic Health, ZIOPHARM Oncology; Travel/expenses: Merck, Spectrum Pharmaceuticals, Lilly, Amgen, Immunomedics, Pfizer, Genentech Puma Biotechnology; Speaker Bureau: Lilly. Institutional funding: Merck, Lilly, BMS, ZIOPHARM Oncology DBP: Research support: BMS, Merck, Brooklyn ImmunoTherapeutics. Advisory Boards: BMS, Merck, Syndax, Nektar, Puma, Nanostring, Genetech, Brooklyn Immunotherapeutics, Sanofi, Biotheranostics, NGMBio, Lilly. Speaker Bureau: Genentech, Novartis.

\section{Patient consent for publication Not applicable.}

Ethics approval This study was approved by the clinical trial of pembrolizumab with capecitabine or paclitaxel was approved by the Providence Portland Medical Center, \#16-001 and Cedars Sinai Medical Center (ID unknown). Collection of earlystage breast cancer specimens was approved by the institutional review board at Providence Portland Medical Center, \#15-162.

Provenance and peer review Not commissioned; externally peer reviewed.

Data availability statement Data are available on reasonable request. The datasets used and/or analyzed during this study are available from the corresponding author on reasonable request.

Supplemental material This content has been supplied by the author(s). It has not been vetted by BMJ Publishing Group Limited (BMJ) and may not have been peer-reviewed. Any opinions or recommendations discussed are solely those of the author(s) and are not endorsed by BMJ. BMJ disclaims all liability and responsibility arising from any reliance placed on the content. Where the content includes any translated material, BMJ does not warrant the accuracy and reliability of the translations (including but not limited to local regulations, clinical guidelines, terminology, drug names and drug dosages), and is not responsible for any error and/or omissions arising from translation and adaptation or otherwise.
Open access This is an open access article distributed in accordance with the Creative Commons Attribution Non Commercial (CC BY-NC 4.0) license, which permits others to distribute, remix, adapt, build upon this work non-commercially, and license their derivative works on different terms, provided the original work is properly cited, appropriate credit is given, any changes made indicated, and the use is non-commercial. See http://creativecommons.org/licenses/by-nc/4.0/.

\section{ORCID iDs}

Brie Chun http://orcid.org/0000-0001-7520-8365

William L Redmond http://orcid.org/0000-0002-2572-1731

David B Page http://orcid.org/0000-0001-9264-4628

\section{REFERENCES}

1 Schmid P, Adams S, Rugo HS, et al. Atezolizumab and nabpaclitaxel in advanced triple-negative breast cancer. N Engl J Med 2018;379:2108-21.

2 Cortes J, Cescon DW, Rugo HS, et al. Pembrolizumab plus chemotherapy versus placebo plus chemotherapy for previously untreated locally recurrent inoperable or metastatic triple-negative breast cancer (KEYNOTE-355): a randomised, placebo-controlled, double-blind, phase 3 clinical trial. Lancet 2020;396:1817-28.

3 Miles D, Gligorov J, André F, et al. Primary results from IMpassion131, a double-blind, placebo-controlled, randomised phase III trial of first-line paclitaxel with or without atezolizumab for unresectable locally advanced/metastatic triple-negative breast cancer. Ann Oncol 2021;32:994-1004.

4 Shah AN, Flaum L, Helenowski I, et al. Phase II study of pembrolizumab and capecitabine for triple negative and hormone receptor-positive, HER2-negative endocrine-refractory metastatic breast cancer. J Immunother Cancer 2020;8:e000173.

5 Mittendorf EA, Zhang $\mathrm{H}$, Barrios $\mathrm{CH}$, et al. Neoadjuvant atezolizumab in combination with sequential nab-paclitaxel and anthracyclinebased chemotherapy versus placebo and chemotherapy in patients with early-stage triple-negative breast cancer (IMpassion031): a randomised, double-blind, phase 3 trial. Lancet 2020;396:1090-100.

6 Schmid P, Cortes J, Pusztai L, et al. Pembrolizumab for early triplenegative breast cancer. N Engl J Med 2020;382:810-21.

7 Postow MA, Manuel M, Wong P, et al. Peripheral T cell receptor diversity is associated with clinical outcomes following ipilimumab treatment in metastatic melanoma. J Immunother Cancer 2015;3:23.

8 Arakawa A, Vollmer S, Tietze J, et al. Clonality of CD4+ blood T cells predicts longer survival with CTLA4 or PD-1 checkpoint inhibition in advanced melanoma. Front Immunol 2019;10:1336.

9 Han J, Duan J, Bai H, et al. TCR repertoire diversity of peripheral $\mathrm{PD}-1^{+} \mathrm{CD} 8^{+} \mathrm{T}$ cells predicts clinical outcomes after immunotherapy in patients with non-small cell lung cancer. Cancer Immunol Res 2020;8:146-54.

10 Hopkins AC, Yarchoan M, Durham JN, et al. T cell receptor repertoire features associated with survival in immunotherapy-treated pancreatic ductal adenocarcinoma. JCl Insight 2018;3

11 Snyder A, Nathanson T, Funt SA, et al. Contribution of systemic and somatic factors to clinical response and resistance to PD-L1 blockade in urothelial cancer: an exploratory multi-omic analysis. PLoS Med 2017;14:e1002309.

12 Zhao J, Huang W, Wu Y, et al. Prognostic role of pretreatment blood lymphocyte count in patients with solid tumors: a systematic review and meta-analysis. Cancer Cell Int 2020;20:15.

13 Manuel M, Tredan O, Bachelot T, et al. Lymphopenia combined with low TCR diversity (divpenia) predicts poor overall survival in metastatic breast cancer patients. Oncoimmunology 2012;1:432-40.

14 Afghahi A, Purington N, Han SS, et al. Higher absolute lymphocyte counts predict lower mortality from early-stage triple-negative breast cancer. Clin Cancer Res 2018;24:2851-8.

15 Wang L, Simons DL, Lu X, et al. Connecting blood and intratumoral $\mathrm{T}_{\text {. }}$ cell activity in predicting future relapse in breast cancer. Nat Immunol 2019;20:1220-30.

16 Liu Y-Y, Yang Q-F, Yang J-S, et al. Characteristics and prognostic significance of profiling the peripheral blood T-cell receptor repertoire in patients with advanced lung cancer. Int $J$ Cancer 2019;145:1423-31.

17 Cohen CJ, Gartner JJ, Horovitz-Fried M, et al. Isolation of neoantigen-specific T cells from tumor and peripheral lymphocytes. $J$ Clin Invest 2015:125:3981-91.

18 Penter L, Dietze K, Ritter J, et al. Localization-associated immune phenotypes of clonally expanded tumor-infiltrating T cells and distribution of their target antigens in rectal cancer. Oncoimmunology 2019;8:e1586409. 
19 Duhen T, Duhen R, Montler R, et al. Co-expression of CD39 and CD103 identifies tumor-reactive CD8 T cells in human solid tumors. Nat Commun 2018;9:2724.

20 Ahmadzadeh M, Pasetto A, Jia L, et al. Tumor-infiltrating human $\mathrm{CD}^{+}$regulatory $\mathrm{T}$ cells display a distinct TCR repertoire and exhibit tumor and neoantigen reactivity. Sci Immunol 2019;4

21 Kagamu H, Kitano S, Yamaguchi O, et al. CD4 ${ }^{+}$T-cell immunity in the peripheral blood correlates with response to anti-PD-1 therapy. Cancer Immunol Res 2020;8:334-44.

22 Bruchard M, Mignot G, Derangère $\mathrm{V}$, et al. Chemotherapy-triggered cathepsin $B$ release in myeloid-derived suppressor cells activates the NLRP3 inflammasome and promotes tumor growth. Nat Med 2013;19:57-64.

23 Vincent J, Mignot G, Chalmin F, et al. 5-Fluorouracil selectively kills tumor-associated myeloid-derived suppressor cells resulting in enhanced T cell-dependent antitumor immunity. Cancer Res 2010;70:3052-61.

24 DeNardo DG, Brennan DJ, Rexhepaj E, et al. Leukocyte complexity predicts breast cancer survival and functionally regulates response to chemotherapy. Cancer Discov 2011;1:54-67.

25 Liechtenstein T, Perez-Janices N, Gato M, et al. A highly efficient tumor-infiltrating MDSC differentiation system for discovery of anti-neoplastic targets, which circumvents the need for tumor establishment in mice. Oncotarget 2014;5:7843-57.

26 Pfannenstiel LW, Lam SSK, Emens LA, et al. Paclitaxel enhances early dendritic cell maturation and function through TLR4 signaling in mice. Cell Immunol 2010;263:79-87.

27 Zhang S, Wang Z, Fan S, et al. Capecitabine can induce T cell apoptosis: a potential immunosuppressive agent with anti-cancer effect. Front Immunol 2021;12:737849.

28 Page DB, Chun B, Pucilowska J, et al. Pembrolizumab (pembro) with paclitaxel (Taxol) or capecitabine (CAPE) as early treatment of metastatic triple-negative breast cancer (mTNBC). JCO 2019;37:1015.

29 Maecker HT, McCoy JP, et al. A model for harmonizing flow cytometry in clinical trials. Nat Immunol 2010;11:975-8.

30 Appay V, Reynard S, Voelter V, et al. Immuno-monitoring of CD8+ $\mathrm{T}$ cells in whole blood versus PBMC samples. J Immunol Methods 2006;309:192-9.

31 Koguchi Y, Gonzalez IL, Meeuwsen TL, et al. A semi-automated approach to preparing antibody cocktails for immunophenotypic analysis of human peripheral blood. J Vis Exp 2016;108:e53485.

32 Maecker HT, McCoy JP, Nussenblatt R. Standardizing immunophenotyping for the human immunology project. Nat Rev Immunol 2012;12:191-200.

33 Carlson CS, Emerson RO, Sherwood AM, et al. Using synthetic templates to design an unbiased multiplex PCR assay. Nat Commun 2013:4:2680

34 Robins HS, Campregher PV, Srivastava SK, et al. Comprehensive assessment of T-cell receptor $\beta$-chain diversity in $\alpha \beta$ T cells. Blood 2009;114:4099-107.

35 Robins H, Desmarais C, Matthis J, et al. Ultra-sensitive detection of rare T cell clones. J Immunol Methods 2012;375:14-19.

36 Yohannes DA, Freitag TL, de Kauwe A, et al. Deep sequencing of blood and gut T-cell receptor $\beta$-chains reveals gluten-induced immune signatures in celiac disease. Sci Rep 2017;7:17977.

37 Kim B-R, Shin J, Guevarra RB, et al. Deciphering diversity indices for a better understanding of microbial communities. J Microbiol Biotechnol 2017;27:2089-93.

38 Emerson RO, Sherwood AM, Rieder MJ, et al. High-throughput sequencing of $\mathrm{T}$-cell receptors reveals a homogeneous repertoire of tumour-infiltrating lymphocytes in ovarian cancer. J Pathol 2013;231:433-40.

39 Zhang H, Liu L, Zhang J, et al. Investigation of antigen-specific T-cell receptor clusters in human cancers. Clin Cancer Res 2020;26:1359-71.
40 Grabchak M, Marcon E, Lang G, et al. The generalized Simpson's entropy is a measure of biodiversity. PLoS One 2017;12:e0173305.

41 Rytlewski J, Deng S, Xie T, et al. Model to improve specificity for identification of clinically-relevant expanded T cells in peripheral blood. PLoS One 2019;14:e0213684.

42 Hughes RA, May MT, Tilling K, et al. Long terms trends in CD4+ cell counts, CD8+ cell counts, and the CD4+. AIDS. 2018;32:1361-7. 06.

43 Serrano-Villar S, Martínez-Sanz J, Ron R, et al. Effects of first-line antiretroviral therapy on the $\mathrm{CD} 4 / \mathrm{CD} 8$ ratio and $\mathrm{CD} 8$ cell counts in CoRIS: a prospective multicentre cohort study. Lancet HIV 2020;7:e565-73.

44 Servaas NH, Zaaraoui-Boutahar F, Wichers CGK. Longitudinal analysis of T-cell receptor repertoires reveals persistence of antigendriven CD4. J Autoimmun 2021;117:102574.

45 Avettand-Fenoel V, Bayan T, Gardiennet E, et al. Dynamics in HIV-DNA levels over time in HIV controllers. J Int AIDS Soc 2019;22:e25221.

46 Verma R, Foster RE, Horgan K, et al. Lymphocyte depletion and repopulation after chemotherapy for primary breast cancer. Breast Cancer Res 2016;18:10.

47 Gustafson CE, Jadhav R, Cao W, et al. Immune cell repertoires in breast cancer patients after adjuvant chemotherapy. JCl Insight 2020;5.

48 Mozaffari F, Lindemalm C, Choudhury A, et al. NK-cell and T-cell functions in patients with breast cancer: effects of surgery and adjuvant chemo- and radiotherapy. Br J Cancer 2007:97:105-11.

49 Tickotsky N, Sagiv T, Prilusky J, et al. McPAS-TCR: a manually curated Catalogue of pathology-associated T cell receptor sequences. Bioinformatics 2017;33:2924-9.

50 Bagaev DV, Vroomans RMA, Samir J. VDJdb in 2019: database extension, new analysis infrastructure and a T-cell receptor motif compendium. Nucleic Acids Research 2019.

51 Krijgsman D, De Vries NL, Andersen MN, et al. The effects of tumor resection and adjuvant therapy on the peripheral blood immune cell profile in patients with colon carcinoma. Cancer Immunol Immunother 2020;69:2009-20.

52 Britanova OV, Putintseva EV, Shugay M, et al. Age-related decrease in TCR repertoire diversity measured with deep and normalized sequence profiling. J Immunol 2014;192:2689-98.

53 Naylor K, Li G, Vallejo AN, et al. The influence of age on T cell generation and TCR diversity. J Immunol 2005;174:7446-52.

54 Boyd SD, Liu Y, Wang C, et al. Human lymphocyte repertoires in ageing. Curr Opin Immunol 2013;25:511-5.

55 Liu WM, Fowler DW, Smith P, et al. Pre-treatment with chemotherapy can enhance the antigenicity and immunogenicity of tumours by promoting adaptive immune responses. $\mathrm{Br} \mathrm{J}$ Cancer 2010;102:115-23.

56 Okoye IS, Xu L, Walker J, et al. The glucocorticoids prednisone and dexamethasone differentially modulate $T$ cell function in response to anti-PD-1 and anti-CTLA-4 immune checkpoint blockade. Cancer Immunol Immunother 2020;69:1423-36.

57 Zacharchuk CM, Merćep M, Chakraborti PK, et al. Programmed T lymphocyte death. cell activation- and steroid-induced pathways are mutually antagonistic. J Immunol 1990;145:4037-45.

58 Apetoh L, Ghiringhelli F, Tesniere A, et al. Toll-like receptor 4dependent contribution of the immune system to anticancer chemotherapy and radiotherapy. Nat Med 2007:13:1050-9.

59 JS D, Min B. Differential requirements of MHC and of DCs for endogenous proliferation of different T-cell subsets in vivo. Proc Nat Acad Sci U S A 2009;106:20394-8.

60 Lowery FJ, Krishna S, Yossef R. Abstract 127: single cell mapping of tumor infiltrating lymphocytes enables neoantigen-reactive $T$ cell identification in metastatic human cancer. Cancer Research $2021 ; 81: 127$ 